\title{
Do multiple sclerosis lesions affect the outcome of magnetic resonance image registration?
}

\author{
Paulo Guilherme de Lima Freire ${ }^{1}$ \\ Ricardo José Ferrari ${ }^{1}$
}

\begin{abstract}
Multiple sclerosis (MS) is a chronic inflammatory demyelinating disease of the central nervous system manifested morphologically by inflammation, demyelination, axonal loss and gliosis. The Magnetic Resonance (MR) imaging, which presents high resolution and good differentiation between brain tissues, is considered the gold standard for the detection and evolution assessment of MS lesions. Fairly recently, a number of automatic image processing systems have been proposed in the literature to help radiologists detect and compute the volume of MS lesions in MR images. Among most of these approaches, registration of multi-contrast MR clinical images (T1/T2/PD/FLAIR) as well as registration of patient's data to anatomical atlases have proved to be essential steps in the neuroimaging processing pipeline to successfully detect and quantitatively assess lesion load in MS. However, despite the importance of image registration and its common use in automatic image processing systems, the effect of MS lesions on the final results of image alignment has not been thoroughly investigated. In this work, image registration techniques, using both affine and deformable transformations, were used to assess if MS lesions (stratified in mild, moderate or severe) had any influence on the registration step. Based on quantitative results obtained using metrics such as Jaccard, Dice and volume overlap, it was verified that MS lesions do not significantly affect the registration process. In the severe lesions case, for instance, the computed Jaccard values for the affine and deformable transformations were, respectively, 0.999 and 0.929 . The only exception was for images with mild MS lesions using deformable registration, in which case the Jaccard value was 0.74 . Also, it was verified that the sole use of affine transformation is very reasonable to correctly align images even if they do have lesions.
\end{abstract}

\section{Introduction}

Multiple Sclerosis is a central nervous system (CNS) disease that affects mainly the young adults population (in the range from 20 to 40 years old). With cause unclear, MS is an inflammatory disease that mainly affects the myelin sheath of the nerve cells in the brain, causing various changes in the patient's muscular function and motion sensibility [19]. Multi-spectral MRI is currently the most used method to diagnose MS because of its high

\footnotetext{
${ }^{1}$ Departamento de Computação, UFSCar, Caixa Postal 676

\{paulo.freire, rferrari\}@dc.ufscar.br
} 
resolution, good soft tissue differentiation and different contrast information (such as T1-, T2-, PD-weighted and FLAIR) [11].

Image registration is the process of transforming different sets of images into a consistent anatomical coordinate system. It is a very common and important step in the pipeline of most image processing systems in neuroimaging, including the ones designed for segmentation of MS lesions in MR images. The process of image alignment is not only used to combine information from anatomical and functional images with the intent to help radiologists detect and diagnose brain diseases, but it is also used to help design automatic image processing techniques. In this case, clinical images are in general aligned to anatomical or probabilistic atlases to provide prior information for the initialization of algorithms that will later be used in a segmentation step. Despite the importance of image registration in neuroimaging and the considerable number of techniques that has been proposed in the literature to perform this task $[3,4,15,18,23]$, large apparent changes in the brain of a patient, due to removal of a portion of it or to the presence of pathologies, are still a significant problem for MR image registration since they may significantly undermine automatic analysis. This is the case, for instance, when performing registration of MR images acquired before and after surgical removal of malignant glioma [12].

In the same manner the absence of brain tissue may affect MR image registration, the presence of pathologies (for instance, MS lesions [27]) may also compromise the alignment of MR images. Crum et al. [10] tackled this problem by using not one reference image, but many images. Their proposed method analyzes the population of images as a whole and uses a composition of well-defined pair-wise registrations to obtain transformations between arbitrary pairs of images. This approach avoids bad results in situations where there are anatomical or pathological differences between the images.

Techniques based on point set (or landmarks) registration using the iterative closest point (ICP) algorithm [2], or more advanced derived ones [7, 21, 13], have also been investigated to overcome the problem of large apparent changes in MR images. Despite the great success of this approach on angiography [26] and retinal image registration [25], the main difficulties with this class of techniques are the detection of reliable and stable landmarks on the images and the design of a unique signature to those points in order to successfully match them on both fixed and moving images before finding the alignment transformation.

Another method to deal with registration of images with missing correspondence regions was proposed by Chitphakdithai and Duncan [6]. In their work, the registration problem is posed in a marginalized maximum a posteriori (MAP) estimation framework in which the transformation and correspondence regions are simultaneously estimated using the expectation-maximization (EM) algorithm. The authors used spatial prior based on principal component analysis (PCA) to guide the selection of an indicator map. However, a problem with this approach is that the estimated indicator map is limited by the training data used to 
build the library of possible maps, which can be problematic with a small training set since it would not be able to capture the full range of indicator possible shapes.

Stefanescu et al. [24] used segmentation algorithms to delineate a pathology and then reduce the weight of the corresponding voxels, consequently reducing their impact on the image registration process. A downside of this approach is that results are as good as the algorithms used for the segmentation task.

Tan et al. [27] conducted a comprehensive study relating the use of registration to correct for rigid misalignment and the presence of MS lesions. MR images from nine different patients with MS lesions were used. Misalignments were purposely introduced in the images and the goal was to assert how accurate would the registration be (using the Normalized Mutual Information - NMI - algorithm) in aligning back the purposely distorted images. According to the authors, the registration remained robust even in the presence of MS lesions. Also, they could not find any trace of evidence that the amount of misalignment introduced in the images had any effect on the outcome. One aspect of their work that needs to be emphasized is that the analysis was done with misalignments caused by rigid transformation only.

In contrast to the work of Tan et al. [27], in this work we assess the influence of MS lesions in MR images by introducing misalignments using affine transformations, i.e., including global scaling and sheering, in addition to rigid transformation. In this case, by applying a known affine transformation to the images and then aligning them back, we simulate a situation where a clinical image is registered with a brain template. Therefore, in addition to the analysis of image-to-image registration for the same patient, we also studied the effect of image-to-atlas registration in the presence of MS lesions. In our experiments, we also investigated two image registration techniques applied to MR images with different lesion loads.

This paper is organized as follows. In Section 2 we present the material and methodology used in this work. Section 3 describes all results and discussions of our investigation. Conclusions about this study are given in Section 4.

\section{Material and methodology}

Image registration using 3-dimensional images consists of finding a spatial transformation $T$ that maps an image $I(x, y, z)$ into another image $J(x, y, z)$ according to a similarity metric $S$. In this process we have a reference image that remains fixed and another image that is transformed in order to provide the best possible mapping (or alignment). Commonly, the reference image is called the fixed image, denoted herein by $F(x, y, z)$, and the image to be transformed is called the moving image and is denoted by $M(x, y, z)$. The result of a 
registration between two 3-dimensional images is a transformation $T(x, y, z): \mathfrak{R}^{3} \rightarrow \mathfrak{R}^{3}$ that maps the $(x, y, z)$ coordinates of one image into another. The transformation is estimated such that the similarity between the fixed image $F$ and the moving image $M$ is maximized. The final transformed image $M^{*}$ is calculated as $M^{*}(x, y, z)=M\left(x-T_{x}(x, y, z), y-\right.$ $\left.T_{y}(x, y, z), z-T_{z}(x, y, z)\right)$ [14]. For the sake of notation simplicity, from here on we will replace the indication of three-dimensional position $(x, y, z)$ by $\vec{x}$.

The following subsections explain in details the techniques that were used in the registration process as well as the images in which they were used.

\subsection{Spatial preprocessing}

MR image acquisition usually does not follow a single protocol, which means that the spacing and/or size of the voxels can be different from one acquisition to another. These spatial differences can greatly affect image registration because they increase dissimilarities between the images. In this work, previous to image registration, a spatial image normalization was conducted to correct differences in origin, voxel size, spacing and axes directions between the images to be registered (fixed and moving). This spatial preprocessing step is necessary to reduce the computational burden of the registration algorithm when trying to realign the raw data provided as inputs. The proposed spatial preprocessing was performed by taking the information (spacing, orientation and voxel size) provided in the header file of the fixed image and applying them to the moving images.

\subsection{Registration modules}

In general, image registration techniques are comprised of four modules that are used during their execution: metric, optimizer, interpolator and transformation. The proposed method shown in Figure 1 consists of finding a transformation $T_{p}(\vec{x})$ to correct the misalignment between the images. The parameters $p$ of the transformation $T$ are obtained in an iterative manner by mapping the voxels from the moving image $M(\vec{x})$ to their corresponding voxels in the fixed image $F(\vec{x})$ so that the similarity metric $S(p \mid F, M, T)$ is minimized.

2.2.1 Metric In this work, the chosen metric was the mutual information (MI) [20, 28], which measures the statistical dependency between two data sets (fixed and moving images) by taking into account the amount of information that one random variable has over another. Mutual information is defined in terms of entropy in the following way [20]:

$$
S(p \mid F, M, T)=M I\left(F, T_{p}(M)\right)=H(F)+H(M \mid u)-H(F, M \mid u),
$$




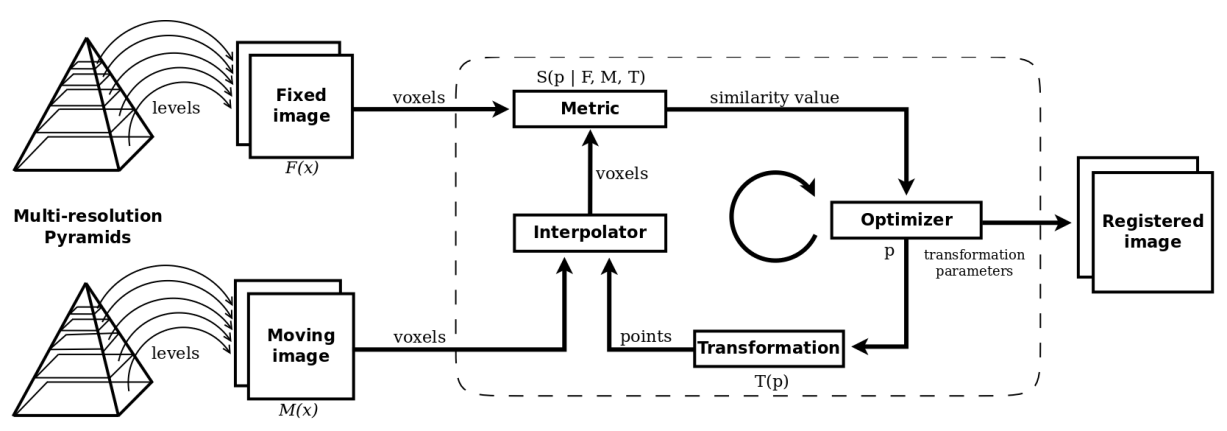

Figure 1. Diagram of the registration modules.

where $H(\cdot)$ is the entropy of a random variable (in this case, images $F$ or $M$ ), which can be calculated from the marginal probability (histogram), $P(\cdot)$, of the images as:

$$
\begin{gathered}
H(F)=-\sum_{m \in F} P(m) \log P(m), \\
H(M \mid u)=-\sum_{n \in M} P(n \mid u) \log P(n \mid u) .
\end{gathered}
$$

In Equations (2) and (3), $m$ and $n$ represent, respectively, voxel intensities in the images $F$ and $M$ and $u$ is the transformation necessary to map $M$ into $F$. Finally, the joint entropy of images $F$ and $M$, which is the last term in Equation (1), is calculated from their joint probability distribution (joint histogram), $P(m, n \mid u)$, as:

$$
H(F, M \mid u)=-\sum_{m \in F, n \in M} P(m, n) \log P(m, n \mid u) .
$$

2.2.2 Optimizer An optimizer, based on the gradient descent method, was used to search for the best set of parameters $p$ that minimizes the similarity function $S$ between images $F$ and $M$ [16]. The method uses a multi-resolution Gaussian pyramid approach in which the levels contain images with different resolutions, as illustrated in Figure 1. The estimation of the parameters starts with the lowest resolution images $\left(1 \times 1 \times 1 \mathrm{~mm}^{3} \times 2^{n}\right.$, where $n=3$ is the number of levels used in this work) from the top level of the pyramids, and the parameters estimated at this level are used as an initial start to the algorithm on the next lower level. This procedure is then repeated until the pyramid bases (full resolution images) are reached. This approach results in a reduction of processing time of the algorithm as well as in an increase 
of the method stability, since the coarse details from the top levels of the pyramids increase the chance of the gradient descent method converge to a point of global minimum, providing, therefore, good parameters estimation in each iteration.

The choice of using the gradient-descent algorithm as an optimizer in our registration framework was made because it is a low computational complexity technique, which is an important feature when processing very large amounts of data. Also, as mentioned previously, the optimization of the parameters is performed using a multi-resolution framework, which minimizes the chances of the gradient-descent algorithm getting trapped in a local minimum and increases the convergence speed.

2.2.3 Interpolator Similarly to the optimizer, a linear interpolator was used in our image registration framework because of its low computational complexity with respect to the number of voxel of a volume. This module is necessary because the mapping of the points from one image into another is performed in the physical coordinate system and, therefore, an interpolator is required to put these points back in their corresponding places in the image pixel grid.

2.2.4 Transformation To test for the influence of MS lesions on the MR image registration, two types of transformations (affine and deformable) were investigated.

An affine transformation is defined as a transformation that maps parallel lines in other lines that are parallel too, but not necessarily keeping their original proportions [17, 9]. In other words, transformations that deal with rotations, translations, scales and shears are affine transformations. Mathematically, an affine transformation is defined as

$$
\vec{x}^{\prime} \mapsto A \vec{x}+b,
$$

where $A$ is a matrix whose coefficients represent the parameters of rotations, scales and shears, whereas $b$ is a column vector that maps translations.

Deformable transformations, on the other hand, are dynamic models that evolve under the influence of internal and external forces [9]. Assuming that there is no affine transformation involved, a deformable transformation consists of finding a mapping of an image $M(\vec{x})$ to an image $F(\vec{x})$ using a deformation field $u(\vec{x})$ [1]. The deformation is defined in the physical image space and provides the positional difference between two given images. In this way, if a feature defined in $F(\vec{x})$ has its equivalent in $M$ in a given position $\vec{y}$, the deformation field $u$ in $\vec{x}$ is computed as

$$
u(\vec{x})=\vec{y}-\vec{x},
$$


and, therefore, it can be applied to deform an image $M$ into an image $F$ by

$$
M_{\text {deformed }}=M(\vec{x}+u(\vec{x})) \text {. }
$$

\subsection{Database}

In this work, synthetic T1-weighted MR images from McGill University BrainWeb MRI simulator [8], with and without MS lesions, were used to assess the results of image registration algorithms. The size of all volumes was $181 \times 181 \times 217$ with $1 \mathrm{~mm}$ isotropic voxel grid in Talairach space, $3 \%$ noise level and $20 \%$ of intensity non-uniformity level. Additional imaging acquisition parameters were: SFLASH $/ 18 \mathrm{~ms} / 10 \mathrm{~ms} / 30^{\circ}$ (scan technique/repetition time (TR)/echo time (TE)/flip angle). MS binary lesion masks provided by BrainWeb were also used in the experiments. In the BrainWeb database, the images with MS lesions are classified based on the MS lesion loads ${ }^{2}$ as mild ( 25 lesions with a total volume of $0.422 \mathrm{~cm}^{3}$ ), moderate ( 74 lesions with a total volume of $3.512 \mathrm{~cm}^{3}$ ) and severe (53 lesions with a total volume of $\left.10.104 \mathrm{~cm}^{3}\right)$.

\subsection{Analysis of MS lesions in the registration process}

To quantitatively assess the influence of MS lesions in the registration process, three pairs of MR images representing a spectrum of lesion loads ranging from mild to severe were used in our experiments. Each image pair was composed of a T1-weighted image and a binary mask (called $B M$ for convenience) with all annotated lesions, as shown in Figure 3(b). Given these images, the following steps were taken in order to assert the influence of lesions in the registration process:

1. Apply a known (synthetic) affine transformation (called trafo1 for convenience) to $T 1_{\text {original }}$ and $B M_{\text {original }}$, generating new images $T 1_{\text {trafo1 }}$ and $B M_{\text {trafo1 }}$.

2. Register image $T 1_{\text {trafo } 1}$ with image $T 1_{\text {original }}$ using affine and deformable transformations, generating two other transformations called trafo $2_{\text {af fine }}$ and trafo $2_{\text {deformable. }}$.

3. Separately apply trafo $2_{\text {affine }}$ and trafo $2_{\text {deformable }}$ to $T 1_{\text {trafo } 1}$ and $B M_{\text {trafol }}$, generating images $T 1_{\text {reg-affine }}, B M_{\text {reg-affine }}, T 1_{\text {reg-deformable }}$ and $B M_{\text {reg-deformable }}$.

4. Quantitatively assess the results through similarity metrics applied to the image pairs (BM original and $\left.B M_{\text {reg-affine }}\right)$ and (BM original and $\left.B M_{\text {reg-deformable }}\right)$.

\footnotetext{
${ }^{2}$ Total volume of brain tissue affected by the MS disease process (as seen on MRI T2-weighted scans).
} 
It is important to note that applying a known transformation to T1-w images and then aligning them back is equivalent to simulating a situation in which we are registering a clinical image with a brain template. This is a very common scenario in image-to-atlas registration and the analysis of influence of MS lesions in this case provides a good overview of how pathologies can affect the registration process. Figure 2 shows the diagram for the four steps described above.

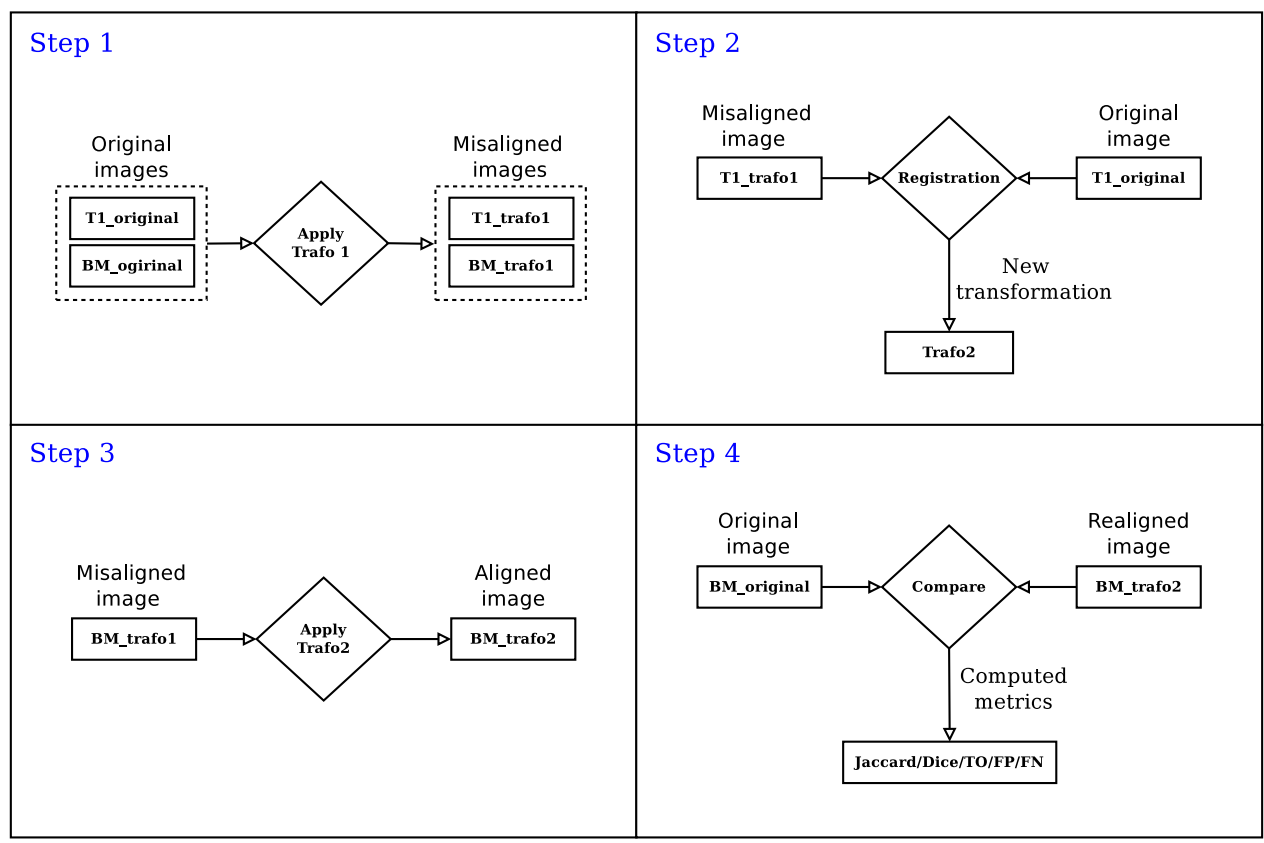

Figure 2. Four steps of the proposed experiment.

Since the transformation applied to $T 1_{\text {original }}$ and $B M_{\text {original }}$ (trafo1) was known, the whole registration process aimed to find an inverse transformation (trafo $2_{\text {af fine }}$ and trafo $2_{\text {deformable }}$ ) that would map $T 1_{\text {trafo1 } 1}$ and $B M_{\text {trafo1 }}$ back to $T 1_{\text {original }}$ and $B M_{\text {original }}$ again. Thus, the lesions influence in the registration could be measured. It is important to note that binary images were not used together with T1 images during the registration. The offsets used in the synthetic transformation (trafo1) were translations of $10 \mathrm{~mm}$ in the $x$-axis, $20 \mathrm{~mm}$ in the $y$-axis, $30 \mathrm{~mm}$ in the $z$-axis, rotation of 15 degrees clockwise and scale of factor 1.2.

The comparison between images $B M_{\text {original }}, B M_{\text {reg-affine }}$ and $B M_{\text {reg-deformable }}$ was conducted to evaluate how well the processed binary masks, i.e., the images resulting 


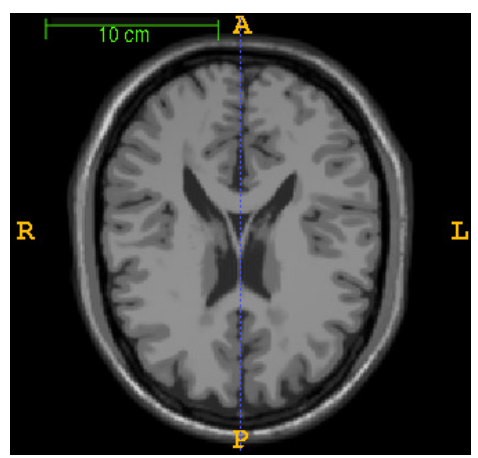

(a)

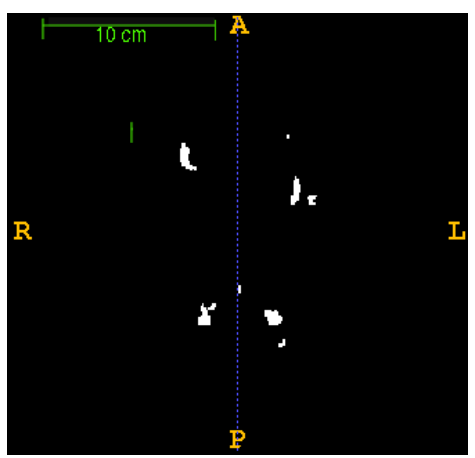

(b)

Figure 3. (a) Axial view of a T1-weighted image with severe MS lesions; (b) binary lesion mask for the image in (a).

from applying the registration algorithm, were aligned compared to the original mask. In this sense, the better the alignment the more robust the process was to the presence of MS lesions.

The same transformation (trafo1) was applied to all three pairs of images (mild, moderate and severe lesions). Since the registration was performed using both affine and deformable transformations for each pair, six results (three for affine transformations and other three for deformable transformations) were obtained. All results were quantitatively assessed by using five different metrics.

In this work, the registration process was executed ten times for each experiment to assess the variability (standard error - SE) of the results. Because the variability of the computed metrics was very low, in this work we have only reported the average values of the metrics.

\subsection{Metrics used to evaluate the results}

After image registration has been completed, five metrics - Total Overlap (TO), Union (Jaccard) [5] [29], Mean Overlap (Dice) [5] [29], False Negatives (FN) and False Positives (FP) rates - were computed from the original and realigned lesion binary masks. These metrics, which are commonly used to compare segmentation results obtained from different techniques, were chosen because they are sensitive to misplacement of the segmentation label (provided by the binary masks) and, therefore, are very useful to quantitatively assess the influence of MS lesions in the registration process.

Taking Figure 4 as a reference, where $I$ is the set of voxels $v$ of the fixed image 
( $\left.B M_{\text {original }}\right)$ and $J$ is the set of voxels $v$ of the registered image ( $B M_{\text {reg-affine }}$ or $B M_{\text {reg-deformable }}$, each metric is calculated as follows:

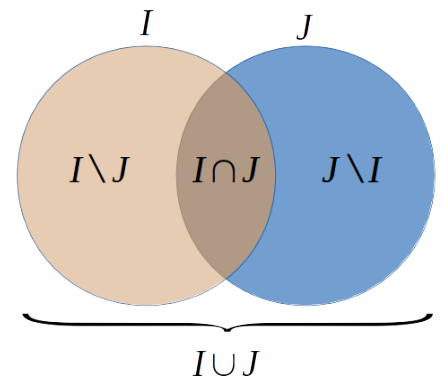

Figure 4. Sets representing two images $(I$ and $J)$.

Total Overlap (TO):

$$
\mathrm{TO}=\frac{\sum_{v}\left|I_{v} \cap J_{v}\right|}{\sum_{v}\left|J_{v}\right|}
$$

Union (Jaccard):

$$
\operatorname{Jaccard}=2 \frac{\sum_{v}\left|I_{v} \cap J_{v}\right|}{\sum_{v}\left|I_{v} \cup J_{v}\right|},
$$

Mean Overlap (Dice):

$$
\text { Dice }=2 \frac{\sum_{v}\left|I_{v} \cap J_{v}\right|}{\sum_{v}\left|I_{v}\right|+\left|J_{v}\right|},
$$

False Negatives (FN) rate:

$$
\mathrm{FN}=\frac{\sum_{v}\left|J_{v} \backslash I_{v}\right|}{\sum_{v}\left|J_{v}\right|}
$$

False Positives (FP) rate:

$$
\mathrm{FP}=\frac{\sum_{v}\left|I_{v} \backslash J_{v}\right|}{\sum_{v}\left|I_{v}\right|}
$$


where $\cap$ and $\cup$ denote the intersection and union operations, respectively.

The closer (or equal) to 1 (one) the metrics Total Overlap (TO), Union (Jaccard) and Mean Overlap (Dice) are, the better. Similarly, the closer (or equal) to 0 (zero) the metrics False Negatives (FN) and False Positives (FP) are, the better.

\section{Results and discussions}

This section presents the results of the quantitative analysis performed to assert the influence of MS-lesions on the image registration. For that, we have conducted two experiments. In the first experiment, we investigated the influence of mild, moderate and severe MS lesions on the image registration using affine transformations. In the second experiment, the same image registration process was repeated using deformable transformations.

Results of the first experiment are shown in Table 1. For the case of mild lesions, it can be noticed that all metrics achieved their best values, indicating that the synthetic distortion initially applied to the original images has been perfectly recovered by the image registration technique. For the moderate lesions, all metrics were slightly bellow to their best values, except for the number of false negatives (FN). Finally, for the severe lesions, the metrics showed an almost perfect recovery from the initial applied synthetic deformation. Although the results obtained by using the affine transformation for all three types of MS lesions are very close to the best values of each metric, it can be noticed in Table 1, there exists a negative correlation between the Jaccard and Dice metrics and the number of the lesions, i.e., the Jaccard and Dice metric values decreases with the increasing of number of lesions.

\section{Affine transformation}

\begin{tabular}{|c|c|c|c|c|c|c|}
\hline Lesion type & Number of lesions $\left(\right.$ volume $\left.\mathrm{cm}^{3}\right)$ & TO & Jaccard & Dice & FN & FP \\
\hline \hline Mild & $25(0.422)$ & 1 & 1 & 1 & 0 & 0 \\
\hline Moderate & $74(3.512)$ & 1 & 0.985 & 0.992 & 0 & 0.015 \\
\hline Severe & $53(10.104)$ & 1 & 0.999 & 0.999 & 0 & 0.001 \\
\hline
\end{tabular}

Table 1. Results of the quantitative analysis of the registration process using affine transformation.

Results of the second experiment (using deformable transformations) are presented in Table 2. In this experiment, the best metric values were obtained for the moderate lesions and the worse numbers for the mild lesions. The results in this case are worse than the ones obtained in the first experiment using affine transformations. Clearly, this fact is more evident when it comes to the case of mild lesions. These differences can be explained by the fact that affine transformations use only global information, whereas deformable transformations 
use local information to decide how the deformation will be; consequently, for the case of deformable transformation, the presence of lesions induce significant local distortions in the registered image, resulting in slightly worse results when compared with values obtained using affine transformation. An alternative to overcome this problem is explored in [22], where the authors suggest that the lesions should be painted in a way they get "camouflaged" in the image. Then the registration would be done as if the image had no lesions whatsoever. After completing the registration, the lesions would be discolored, avoiding any influence they could have in the registration process.

\section{Deformable transformation}

\begin{tabular}{|c|c|c|c|c|c|c|}
\hline Lesion type & Number of lesions $\left(\right.$ volume $\left.\mathrm{cm}^{3}\right)$ & TO & Jaccard & Dice & FN & FP \\
\hline \hline Mild & $25(0.422)$ & 0.857 & 0.744 & 0.854 & 0.143 & 0.115 \\
\hline Moderate & $74(3.512)$ & 1 & 0.973 & 0.987 & 0 & 0.027 \\
\hline Severe & $53(10.104)$ & 0.989 & 0.929 & 0.963 & 0.011 & 0.061 \\
\hline
\end{tabular}

Table 2. Results of the quantitative analysis of the registration process using deformable transformation.

Analyzing all results presented in Tables 1 and 2, it can be observed that the registration was successful in both affine and deformable approaches, with the exception for the case of using deformable transformation applied to images with mild lesions. However, the lower Jaccard/Dice values should be considered with the caveat that these metrics can be greatly influenced when assessing similarity between small objects.

To serve as a normal control case for comparison with the results obtained from the experiments designed to assess the influence of the MS lesions on the image registration procedure, the same technique described in section 2.4 was applied to a healthy brain image. Similarly to the MS lesions in the pathological images, the corpus callosum and its respective binary mask were used to assess the effect of image registration on a normal brain image. Figure 5 shows a T1-weighted sagittal image of the brain and its respective corpus callosum binary mask.

After the registration has been completed, the similarity metrics were computed from the re-aligned corpus callosum structure. For the sake of comparison, results of this test with a normal brain are presented, along with the previous results of the experiments with MS-lesions, in Tables 3 (affine transformation) and 4 (deformable transformations). 


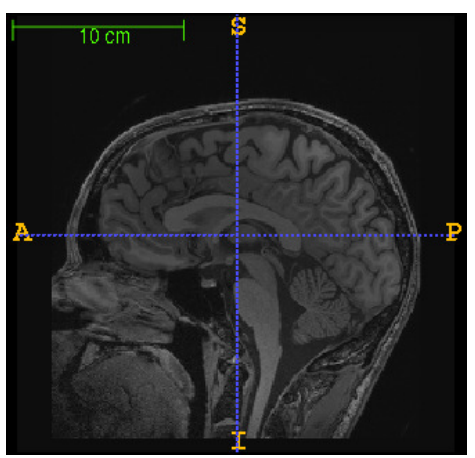

(a)

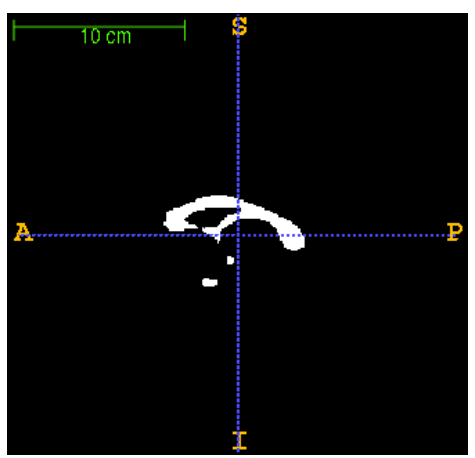

(b)

Figure 5. (a) T1-weighted image of a healthy brain and (b) its respective corpus callosum binary mask.

Affine transformation

\begin{tabular}{|c|c|c|c|c|c|}
\hline & TO & Jaccard & Dice & FN & FP \\
\hline \hline Normal Brain - Corpus Callosum & 1 & 1 & 1 & 0 & 0 \\
\hline Mild lesions & 1 & 1 & 1 & 0 & 0 \\
\hline Moderate lesions & 1 & 0.985 & 0.992 & 0 & 0.015 \\
\hline Severe lesions & 1 & 0.999 & 0.999 & 0 & 0.001 \\
\hline
\end{tabular}

Table 3. Comparison of results of image registration using affine transformation.

Deformable transformation

\begin{tabular}{|c|c|c|c|c|c|}
\hline & TO & Jaccard & Dice & FN & FP \\
\hline \hline Normal Brain - Corpus Callosum & 0.997 & 0.992 & 0.996 & 0.003 & 0.005 \\
\hline Mild lesions & 0.857 & 0.744 & 0.854 & 0.143 & 0.115 \\
\hline Moderate lesions & 1 & 0.973 & 0.987 & 0 & 0.027 \\
\hline Severe lesions & 0.989 & 0.929 & 0.963 & 0.011 & 0.061 \\
\hline
\end{tabular}

Table 4. Comparison of image registrations results using deformable transformation.

As it can be verified from Tables 3 and 4, the results of image registration obtained for a healthy brain are very close to those of images of brains with MS lesions, excepted for the case of the image with mild lesions using deformable transformation. 


\section{Conclusions}

This paper presented a set of experiments to quantitatively assess the influence of MS lesions in the image registration process. As discussed in previous sections of this paper, image registration is a very important step of the pipeline of many image processing systems in neuroimaging. Despite this fact, based on the authors knowledge there is only one work presented in the literature [27] to discuss the influence of MS lesions on the results of image registration. Therefore, in this work we have conducted a few experiments to assess the influence of the MS lesions on the MR image registration. Our experiments used affine and deformable transformations and MR images containing different lesion loads (mild, moderate and severe). Five metrics were used to measure the differences in the original and realigned binary lesions masks. Based on quantitative results of our experiments, we can conclude that, except for the case of MR images with mild lesions processed with deformable registration, the presence of MS lesions in the images have not affected the image registration process. Despite the low values for the Jaccard and Dice metrics, it should be noticed that these metrics can be influenced if the objects being evaluated are too small. Finally, we can also conclude that the use of affine transformations, which are much simpler and faster than deformable transformations, are an appropriated choice to register MR images containing MS lesions.

\section{Acknowledgments}

The authors would like to thank Fundação de Amparo à Pesquisa do Estado de São Paulo (FAPESP) for the financial support of this research - processes numbers 2012/16964-6, 2012/03100-3, and 2014/00019-6.

\section{References}

[1] B. Avants, N. Tustison, and G. Song. Advanced Normalization Tools (ANTS). University of Pennsylvania, 2009.

[2] P.J. Besl and N.D. McKay. A method for registration of 3-D shapes. IEEE Transactions on Pattern Analysis and Machine Intelligence, 14(2):239-256, 1992.

[3] G. Bonniaud, A. Isambert, F. Dhermain, A. Beaudré, I. Ferreira, M. Ricard, and D. Lefkopoulos. Image registration for radiation therapy: practical aspects and quality control. Cancer/Radiothérapie, 10(5):222-230, 2006.

[4] G.M. Cattaneo, M. Reni, G. Rizzo, P. Castellone, G.L. Ceresoli, C. Cozzarini, A.J. Ferreri, P. Passoni, and R. Calandrino. Target delineation in post-operative radiotherapy of brain gliomas: interobserver variability and impact of image registration of 
$\mathrm{mr}$ (pre-operative) images on treatment planning ct scans. Radiotherapy and Oncology, 75(2):217-223, 2005.

[5] H.H. Chang, A.H. Zhuang, D.J. Valentino, and W.C. Chu. Performance measure characterization for evaluating neuroimage segmentation algorithms. Neuroimage, 47(1):122$135,2009$.

[6] N. Chitphakdithai and J.S. Duncan. Pairwise registration of images with missing correspondence due to resection. In Proceedings of the 2010 IEEE International Symposium on Biomedical Imaging: From Nano to Macro, pages 1025-1028, Rotterdam, The Netherlands, April 2010.

[7] H. Chui and A. Rangarajan. A new point matching algorithm for non-rigid registration. Computer Vision and Image Understanding, 89(2):114-141, 2003.

[8] C.A. Cocosco, V. Kollokian, and A. Evans. Brainweb: Online interface to a 3D MRI simulated brain database. NeuroImage, 5(4):425, 1997.

[9] ITK Software Consortium. Insight Into Images - Principles and Practice for Segmentation, Registration, and Image Analysis. A K Peters, 2004.

[10] W.R. Crum, M. Modo, A.C. Vernon, G.J. Barker, and S.C. Williams. Registration of challenging pre-clinical brain images. Journal of Neuroscience Methods, 216(1):62-77, 2013.

[11] W. Gibby. Basic principles of magnetic resonance imaging. Neurosurgery Clinics of North America, 16(1):1-64, 2005.

[12] A. Gooya, K. Pohl, M. Bilello, L. Cirillo, G. Biros, R.E. Melhem, and C. Davatzikos. Glistr: Glioma image segmentation and registration. IEEE Transactions on Medical Imaging, 31(10):1941-1954, 2012.

[13] H. Guo and A. Rangarajan. Diffeomorphic point matching with applications in biomedical image registration, international journal of tomography and statistics. International Journal of Tomography and Statistics, 15(F10):42-57, 2010.

[14] D.L.G. Hill, P.G. Batchelor, M. Holden, and D.J. Hawkes. Medical image registration. Physics in Medicine and Biology, 46(3):R1, 2001.

[15] E.P. Jansen, L.G. Dewit, M. van Herk, and H. Bartelink. Target volumes in radiotherapy for high-grade malignant glioma of the brain. Radiotherapy and Oncology, 56(2):151$156,2000$. 
[16] S. Klein, J.P. Pluim, and M. Staring. Adaptive stochastic gradient descent optimisation for image registration. International Journal of Computer Vision, 81(3):227-239, March 2009.

[17] J.B.A. Maintz and M.A. Viergever. A survey of medical registration. Medical Image Analysis, 2(1):1-36, 1998.

[18] A. Mang, C. Camara, G.B. Caseiras, W. Crum, J.A. Schnabel, T.M. Buzug, J. Thornton, H.R Jäger, and D.J. Hawkes. Image registration of structural and physiological MR images of abnormal anatomy. In Thorsten M. Buzug, Dietrich Holz, Jens Bongartz, Matthias Kohl-Bareis, Ulrich Hartmann, and Simone Weber, editors, Advances in Medical Engineering, volume 114, pages 211-216. Springer Berlin Heidelberg, 2007.

[19] J.H. Noseworthy, C. Lucchinetti, M. Rodriguez, and B.G. Weinshenker. Multiple Sclerosis. The New England Journal of Medicine, 343(13):938-952, 2000.

[20] A. Papoulis. Probability, Random Variables, and Stochastic Processes. McGraw-Hill, Inc., 1991.

[21] A.S. Roy, A. Gopinath, and A. Rangarajan. Deformable density matching for 3D nonrigid registration of shapes. Medical Image Computing and Computer-Assisted Intervention, 10(Pt 1):942-949, 2007.

[22] M. Sdika and D. Pelletier. Nonrigid registration of multiple sclerosis brain images using lesion inpainting for morphometry or lesion mapping. Human Brain Mapping, 30(4):1060-1067, 2009.

[23] A. Sotiras, C. Davatzikos, and N. Paragios. Deformable medical image registration: A survey. IEEE Transactions on Medical Imaging, 32(7):1153-1190, 2013.

[24] R. Stefanescu, O. Commowick, G. Malandain, P.Y. Bondiau, N. Ayache, and X. Pennec. Non-rigid atlas to subject registration with pathologies for conformal brain radiotherapy. In Medical Image Computing and Computer-Assisted Intervention, volume 3216 of MICCAI, pages 704-711, Saint-Malo, France, September 2004.

[25] C.V. Stewart, C.L. Tsai, and B. Roysam. The dual-bootstrap iterative closest point algorithm with application to retinal image registration. IEEE Transactions on Medical Imaging, 22(11):1379-1394, 2003.

[26] J.S. Suri and S. Laxminarayan. Angiography and Plaque Imaging: Advanced Segmentation Techniques. CRC Press, 2003.

[27] I.L. Tan, R.A. Schijndel, M.A. Walderveen, M. Quist, R. Bos, P.J. Pouwels, P. Desmedt, H.J. Adèr, and F. Barkhof. Magnetic resonance image registration in multiple sclerosis: 
Comparison with repositioning error and observer-based variability. Journal of Magnetic Resonance Imaging, 15(5):505-510, 2002.

[28] B. Zitová and J. Flusser. Image registration methods: a survey. Image and Vision Computing, 21(11):977-1000, 2003.

[29] K.H. Zou, S.K. Warfield, A. Bharata, C.M. Tempany, M.R. Kaus, and S.J. Haker. Statistical validation of image segmentation quality based on a spatial overlap index. Academic Radiology, 11(2):178-189, 2004. 